\title{
Single molecule fluorescence spectroscopy of mutants of the Discosoma red fluorescent protein DsRed
}

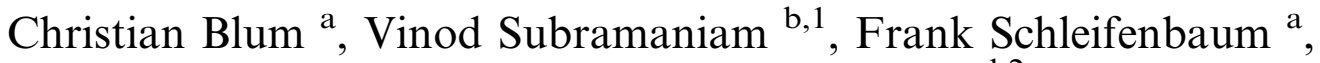 \\ Frank Stracke $^{\mathrm{a}}$, Brigitte Angres ${ }^{\mathrm{c}}$, Alexey Terskikh ${ }^{\mathrm{d}, 2}$, A.J. Meixner ${ }^{\mathrm{a}, *}$ \\ ${ }^{a}$ Physical Chemistry I, University of Siegen, Adolph Reichwein Strasse 2, D-57068 Siegen, Germany \\ ${ }^{\mathrm{b}}$ Department of Molecular Biology, Max Planck Institute for Biophysical Chemistry, D-37077 Göttingen Germany \\ ${ }^{\mathrm{c}}$ BD Biosciences Clontech, 1020 East Meadow Circle, Palo Alto, CA 94303, USA \\ d School of Medicine, Beckman Center, Stanford University, Stanford CA 94305, USA
}

Received 13 March 2002

\begin{abstract}
We studied the emission of mutants of the red fluorescent protein DsRed by room temperature single molecule fluorescence spectroscopy. Bulk samples of the DsRed variant E8 show mixed green and red fluorescence of equivalent intensities individually spectrally similar to arrested green and mature red fluorescent forms of DsRed. Investigations at the single molecule level indicate that, like DsRed, E8 is not monomeric at single molecule concentrations. The entities visualized are composed of green and red emitting proteins without a fixed ratio of green to red fluorescing units. We find indications for only weak, if any, fluorescence resonance energy transfer (FRET) between red and green chromophores within one E8 entity. (c) 2002 Elsevier Science B.V. All rights reserved.
\end{abstract}

\section{Introduction}

The new red fluorescent protein drFP583 (commercially available as DsRed) isolated from the coral Discosoma sp. [1] has significantly en-

\footnotetext{
${ }^{*}$ Corresponding author. Fax: +49-271-7402805.

E-mail address: meixner@chemie.uni-siegen.de (A.J. Meixner).

${ }^{1}$ Present address: Advanced Science and Technology Laboratory, AstraZeneca R\&D Charnwood, Bakewell Road, Loughborough LE11 5RH, United Kingdom.

${ }^{2}$ Corresponding author concerning the mutation of the proteins. E-mail: terskikh@burnham.org. Present address: The Burnham Institute, 10901 North Torrey Pines Road, La Jolla, CA 92037, USA.
}

hanced the palette of fluorescent proteins available for use as markers for visualization of proteins and for detection of protein-protein interactions. The red-shifted absorption and emission spectra of DsRed (relative to the furthest red-shifted variant of the Aequoria GFP) make possible multicolor labeling applications as well as enable the use of DsRed as a likely acceptor for FRET studies with the conventional GFPs as donors [2,3]. However, the use of DsRed in FRET studies is complicated by the maturation behavior into the red form of the protein via a green fluorescing intermediate [3-5] and exceedingly complex photophysics of the molecule [6-11]. The structure of the protein has been solved by X-ray crystallography, and 
possesses the same basic fold as the Aequoria GFPs [12,13].

DsRed has been extensively mutated to yield variants with modified photophysical properties $[5,14,15]$. Recently, mutants of the protein have been prepared which exhibit emission spectra (Fig. 1) similar to those of the immature green form (AG4; with amino acid replacements Val71Met, Val105Ala, Ser197Thr) [16], the red and green forms together (E8; Asn42His), and a well characterized time-dependent mutant initially exhibiting green fluorescence which changes to red fluorescence with time (E5; Val105Ala, Ser197Thr)
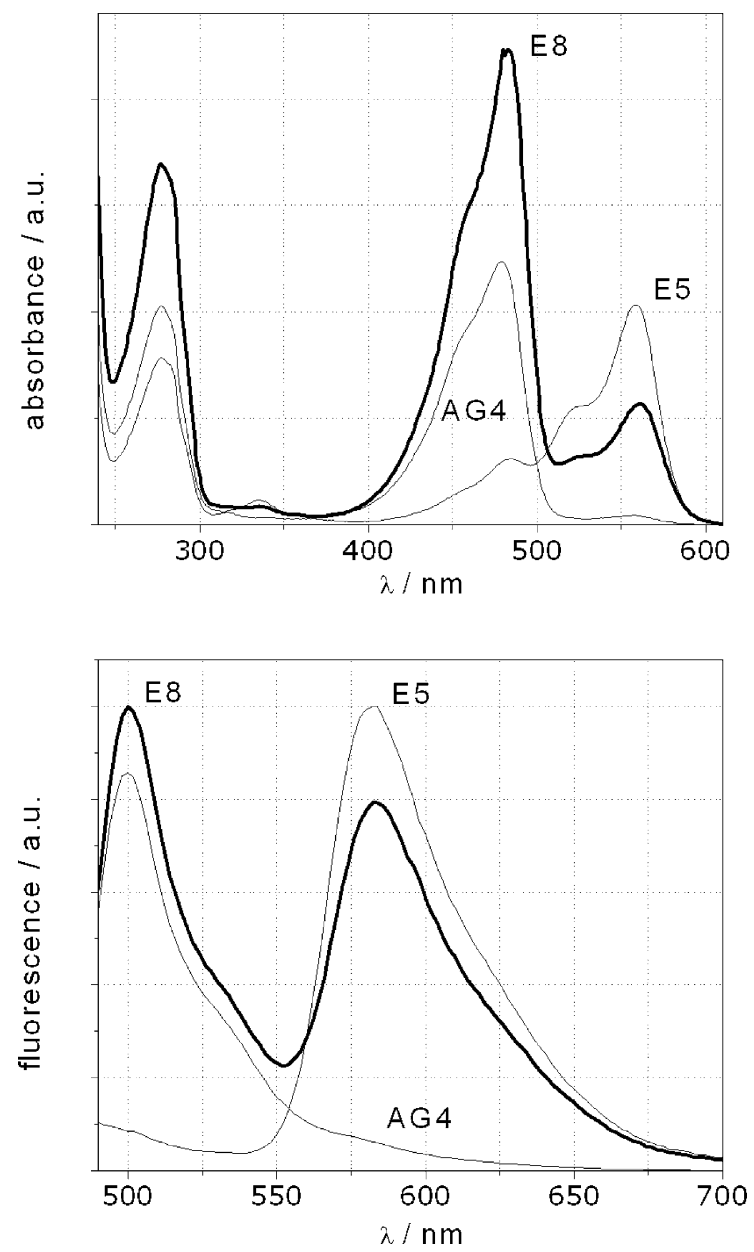

Fig. 1. Ensemble absorbance and fluorescence spectra of the DsRed mutants AG4, E5 and E8 in 100 mM Tris, pH 8.5, 100 $\mathrm{mM} \mathrm{NaCl}$ buffer solution. For emission spectra samples were excited at $\lambda_{\mathrm{ex}}=480 \mathrm{~nm}$.
[14]. To better understand the photophysics of this class of molecules, we investigated the different variants of DsRed on a single-molecule level. It is widely assumed that DsRed forms a tetramer in solution [5], which raises questions about the composition of the tetramer, i.e., the ratio of monomer subunits with green and red chromophores. Spectrally resolved single-molecule studies can reveal if these proteins form homo- or heterooligomers, and should be able to detect FRET between unlike chromophores in a hetero-oligomer. The E8 variant of DsRed, exhibiting both green and red fluorescence of comparable intensities (Fig. 1), exhibits very rapid maturation of the chromophore [17], and provides a photophysically stable system to study the distribution of red and green chromophores. Information about the monomer stoichiometry and photophysics gleaned from studies of this mutant may assist in rational design of further DsRed mutants with tailored fluorescence properties.

\section{Materials and methods}

The mutants AG4 (Val71Met, Val105Ala, Ser197Thr), E5 (Val105Ala, Ser197Thr) and E8 (Asn42His) were generated in a previously performed random mutagenesis of DsRed described in [14]. The E8 mutant, whose characteristics have not been described before by us, exhibits rapid chromophore formation (data not shown) and green and red emissions of similar intensities (Fig. 1). Asn 42 has an important structural role in DsRed, serving to position the Gln66 side chain which forms an integral part of the chromophore [13]. Mutations of this residue, including Asn42His, have been independently found by others through similar mutagenesis approaches $[15,17$, 18], and yield DsRed variants with a higher proportion of green emission than in the wild-type.

The single molecule studies were performed with a classical scanning stage confocal fluorescence microscopy setup (for details see [19]). A single mode argon-ion laser at $488 \mathrm{~nm}$ served as the excitation light source. The excitation intensity was on the order of $1 \mathrm{~kW} / \mathrm{cm}^{2}$, which is well below the fluorescence saturation intensity [8]. Sample 
preparation was accomplished by preparing a dilution series of the fluorescent proteins in $100 \mathrm{mM}$ $\mathrm{Na}$ cacodylate buffer, $\mathrm{pH}$ 7.5. The diluted solution was then mixed with cacodylate buffered polyvinylalcohol and spincoated onto a microscopy cover slide. Imaging of the matrix alone confirmed that fluorescent impurities were negligible. Fluorescence intensity images were obtained by raster scanning the samples and detecting the emission intensity with an avalanche photo diode. From these images distinct fluorescent spots were chosen for spectrally resolved investigations. These fluorescence spots do not necessarily originate solely from tetramers, but from a distribution of species from monomer to tetramer. Due to the forming of these aggregates we will not speak of 'single molecules' but of 'single entities' or 'single units'. Integration times for single entity emission spectra were $1 \mathrm{~s}$ and $3 \mathrm{~s}$ per spectrum, with a dead time between two subsequent spectra of $\sim 25 \mathrm{~ms}$.

\section{Results and discussion}

To estimate if the vast majority of the proteins were still tetrameric at the low concentrations typically used for single molecule studies (about $\left.10^{-9} \mathrm{~mol} / \mathrm{l}\right)$ we measured the fluorescence anisotropy as a function of concentration $\left(10^{-4}-10^{-7}\right.$ $\mathrm{mol} / \mathrm{l}$, data not shown). An increase in the anisotropy is visible, suggesting that partial dissociation takes place at nanomolar concentrations. Thus, in our experimental situation, we do not expect to sample solely tetramers, but a distribution of species from monomer to tetramer.

Fig. 2. Typical single molecule spectra of E5 (top panel), AG4 (bottom panel) and E8 (panels in between). Single entity emission spectra of E5 and AG4 showed almost exclusively only one spectral band at about $580 \mathrm{~nm}$ and 500 , respectively. The observed E8 entities exhibited pure red and pure green fluorescence as well as mixed green and red emission in various intensity ratios. The red and green emission of E8 matches the emission of E5 and AG4. As most of the investigated E8 entities show mixed green and red fluorescence, it can be concluded that E8 forms hetero-entities of statistical composition of proteins embodying green and red emitting chromophores. Further the green fluorescence is not effectively quenched by FRET to the red emitting chromophore.
In Fig. 2 we present various single entity fluorescence spectra from the different DsRed variants. Spectra of E5 showed almost exclusively one spectral band at about $580 \mathrm{~nm}$ (Fig. 2, top panel), and those of AG4 one band at about $500 \mathrm{~nm}$ (Fig. 2 , bottom panel), as expected from ensemble measurements. However, a small number of single

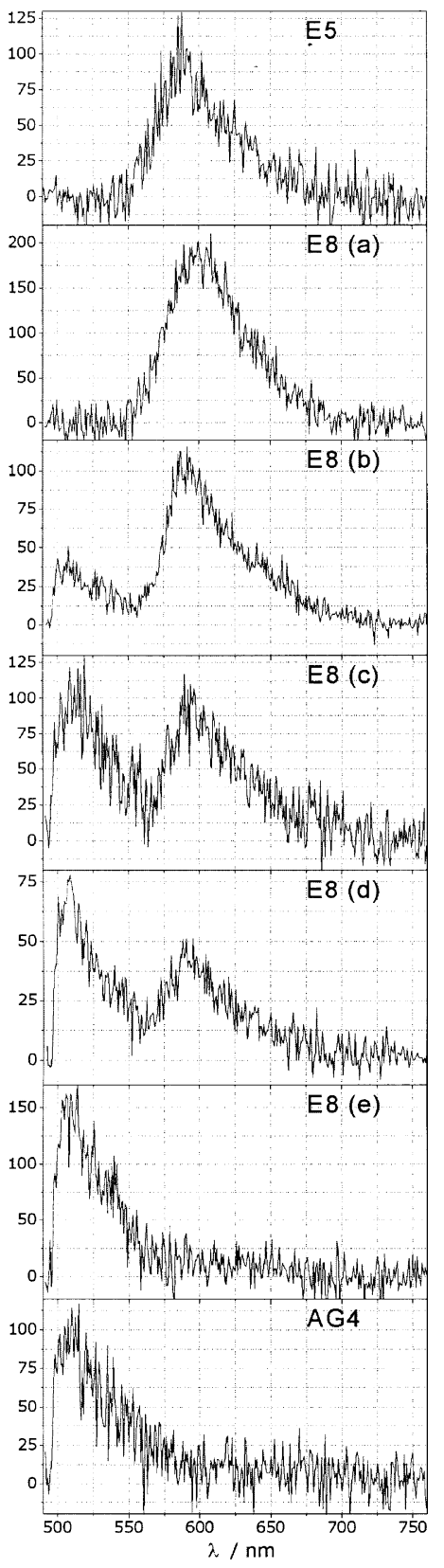


fluorescing units of both mutants showed mixed red and green fluorescence at the same time, suggesting the existence of green chromophores in E5 and red chromophores in AG4, as well as the formation of hetero-entities in E5 and AG4. Ensemble absorption and emission spectra are consistent with this observation; there is a small but finite absorbance at $560 \mathrm{~nm}$, corresponding to the red fluorescing species in the AG4 protein, and a small green component in E5 (Fig. 1).

We performed a simple spectral analysis on 302 units that we determined to be single E8 entities. The single entity emission spectra of E8 exhibited a wide range of green to red intensity ratios, indicating that there is no defined stoichiometry of green and red emitters forming one unit. We found pure red and pure green emitting units (Fig. 2, E8(a), E8(e)) as well as examples with mixed spectra of different band intensity ratios, as shown in Fig. 2, E8(b-d).

As depicted in Fig. 2 the pure red emission of E8 corresponds to that of E5 in spectral shape and position. The pure green emission observed for some E8 units corresponds with the AG4 spectra in shape and position (Fig. 2). Most of the observed single molecule spectra of E8 show both green and red emission bands, suggesting that these entities are composed of red as well as green emitters. As we find a broad distribution of green to red intensity ratios we conclude that the units observed are randomly composed of proteins with green and red chromophores, and that the ratio of red to green fluorescing proteins is not fixed in E8 entities. As the proteins forming one unit have different photophysical parameters and excitation rates, the ratio of band intensities does not allow us to define a stoichiometry for the observed unit. Table 1 gives the number of molecules exhibiting red, green, or mixed red and green fluorescence.

Recent reports in the literature have demonstrated FRET in the wild-type DsRed molecule

Table 1

\begin{tabular}{lc}
\hline Spectral appearance & Number of entities \\
\hline Pure green & 42 \\
Pure red & 83 \\
Red and green & 177 \\
\hline
\end{tabular}

[20] and suggest that the ratio between green and red fluorescing proteins in DsRed is near 1:1 [5,11]. The near absence of green fluorescence was explained with almost total transfer of the excitation energy from the green emitter to a red fluorescing chromophore (FRET) within one tetramer unit. The observed weak green fluorescence in DsRed should originate mainly from tetramers without any red emitting chromophore. If E8 forms heterotetramers a similar mechanism could be expected. As we find predominantly mixed single molecule spectra, two conclusions can be drawn. First, E8 indeed forms hetero-oligomers and second, the green fluorescence is not effectively quenched by FRET to the red emitting chromophore. The finding of non-monomer entities at single molecule level is consistent with results of Lounis et al. [8] and Cotlet et al. [9] who concluded from DsRed single entity time trajectories that there are still tetramers at single molecule concentrations.

While observing the evolution of single entity fluorescence we often found characteristic changes in the green to red fluorescence intensity ratios. If the red and green emitting chromophores form an equilibrium, these changes may occur from individual chromophores switching between red and green fluorescing forms. Preliminary temperature dependent bulk measurements on E8 indeed show a change in the green to red fluorescence intensity ratio, but the fluorescence lifetimes also change correspondingly. We therefore think that the observed single entity spectral behavior is mainly due to successive bleaching of the chromophores within one E8 entity. When studying the possible correlation of the green and red emission in E8, the bleaching behavior of the observed single units is of particular interest. In case of FRET the bleaching of the acceptor chromophore should result in a corresponding rise in the donor fluorescence. In our case, the disappearance of the red emission from a mixed spectrum should lead to a rise in green emission, if this was indeed quenched by energy transfer. If the donor bleaches before the acceptor, a loss in acceptor fluorescence intensity should occur, assuming that the acceptor is excited through the donor by energy transfer.

We observed 75 entities where the mixed green and red fluorescence changed to pure green emis- 
sion. Of these, in 70 cases there was no significant rise in the green emission band intensity, whereas in 5 cases we did observe an increase (see Table 2). In Fig. 3a example of vanishing red emission without a change in the green emission is given. At first mixed emission is detected, while in the second spectrum the red band is completely missing without any change in the green emission. Clearly there was no detectable dequenching of the green fluorescence. During the third detection interval the green chromophore bleaches.

A total of eight entities showed a change from mixed green and red to pure red emission, two with a drop in red fluorescence, and six without. A complete drop of the green fluorescence band without affecting the red fluorescence is depicted in Fig. 3b. The first and third spectrum show mixed fluorescence whereas in spectrum two and four hardly any green fluorescence can be seen. However, the intensity, shape, and spectral position of the red fluorescence band remain the same in all spectra. Obviously the red and green fluorescence is uncorrelated, thus the red emitter is not an efficient FRET acceptor.

Finally we observed four molecules with emission changing from pure red to pure green from one spectrum to the next. In these cases the green chromophore might have been effectively quenched by FRET before the bleaching of the red chromophore. For four molecules we detected a dark period with no emission after the pure red fluorescence vanished and before the pure green fluorescence appeared. In these cases at least one

Table 2

\begin{tabular}{ll}
\hline Spectral appearance & Number of entities \\
\hline $\begin{array}{l}\text { Mixed fluorescence to pure green, } \\
\text { no green dequenching }\end{array}$ & 70 \\
$\begin{array}{l}\text { Mixed fluorescence to pure green, } \\
\text { possible green dequenching }\end{array}$ & 5 \\
$\begin{array}{l}\text { Mixed fluorescence to pure red, drop } \\
\text { in red fluorescence }\end{array}$ & 2 \\
$\begin{array}{l}\text { Mixed fluorescence to pure red, } \\
\text { no drop in red fluorescence }\end{array}$ & 6 \\
$\begin{array}{l}\text { Pure red to pure green, no possible } \\
\text { dequenching of green, dark time } \\
\text { before green arises }\end{array}$ & 4 \\
$\begin{array}{l}\text { Pure red to pure green, possible } \\
\text { dequenching of green }\end{array}$ & 4 \\
Pure green to pure red & 1 \\
\hline
\end{tabular}
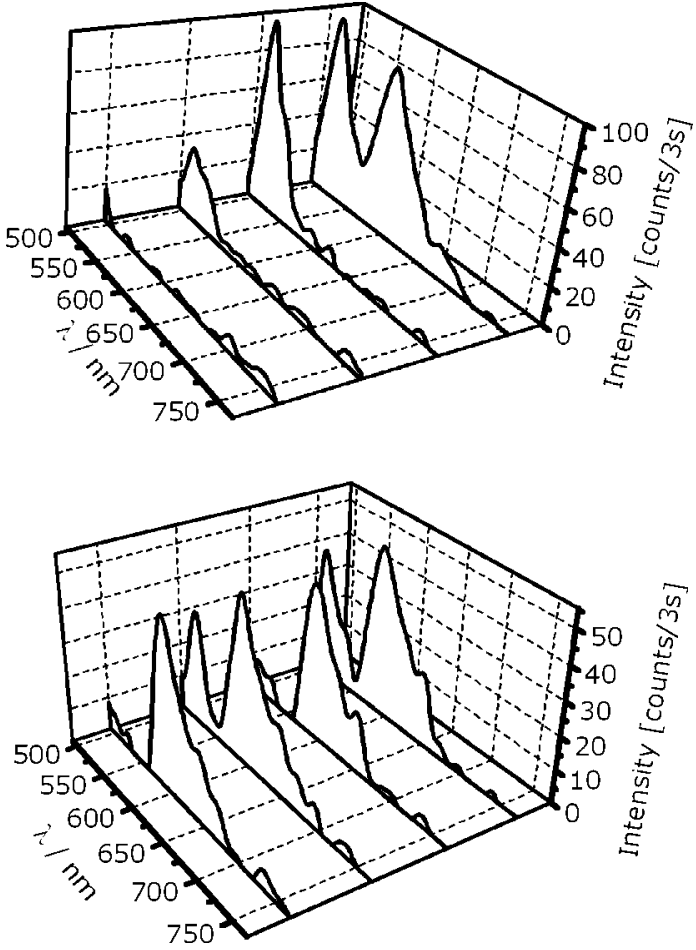

Fig. 3. Spectral sequences of single E8 entities showing spectral dynamics. For better visualization, the spectra were smoothed by convolution with a Gaussian $(\sigma=3 \mathrm{~nm})$. (a) Mixed red and green emission changing to pure green emission. The bleaching of the red emission does not change the green emission. Thus the green emitter was not effectively quenched by FRET to the red emitter. (b) Mixed green and red emission changing twice to pure red emission whereas the spectral position and intensity stays constant. The vanishing of the green fluorescence does not affect the red fluorescence, suggesting that the red emitter is not an effective FRET acceptor.

other phenomenon, namely the change of the green emitter from a dark state into a fluorescing state, can be invoked in lieu of FRET to explain the observation. Only once in a total of 302 cases a change from pure green to pure red fluorescence was detected (all dynamics/bleaching behavior, see Table 2).

In Fig. 4 we present a single entity spectral sequence where mixed green and red fluorescence is visible for the whole observation window, but where the green to red ratio changes substantially with time. The evolution of the band maxima intensities (Fig. 4b) and the correlation diagram for the green and red band maxima intensities (Fig. 4c) are also shown. Due to the noise associated 
(a)

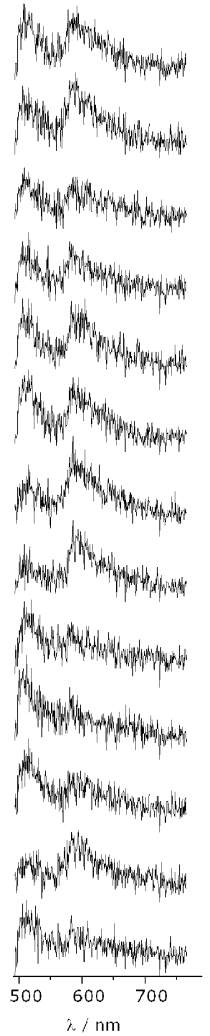

(b)

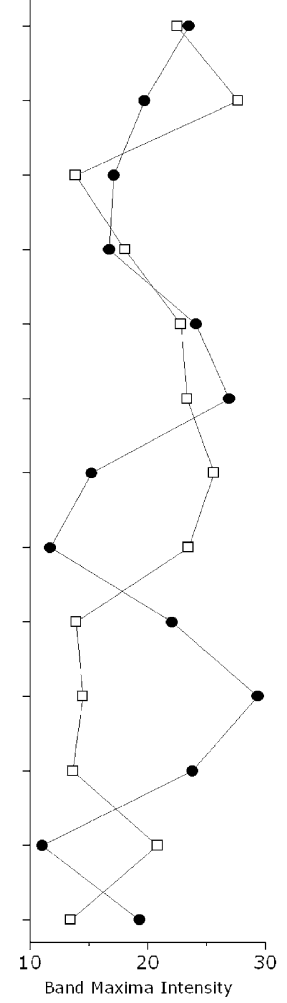

(c)

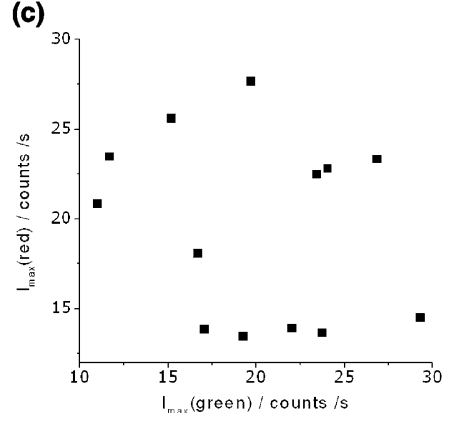

Fig. 4. Fluctuating green to red intensity ratios of a single E8 entity. (a) Shown is the observed emission (1s integration time per spectrum), (b) the evolution of the band maxima intensities (open symbols - red emission band; filled symbols - green emission band) and (c) the correlation plot of the intensities of the band maxima. As no correlation between the evolution of the green and red emission maxima can be seen, the observed changes are not due to varying FRET efficiency between the green and red chromophore.

with the data it was necessary to smooth the data to obtain these intensities methodically. The spectra were smoothed by convolution (sliding

mean) with a Gaussian ( $\sigma=3 \mathrm{~nm})$ followed by determination of the intensities of the band maxima. If the change in the band maxima ratios originates from a varying FRET efficiency, an anti-correlation between the green and the red emission intensity should be expected. Yet it is clearly seen that a decrease in red emission does not systematically lead to an increase in green fluorescence and vice versa. That there is no correlation between the evolution of the red and green band intensity maxima can also clearly been seen from the correlation diagram (Fig. 4c).

An overwhelming majority of these single molecule observations show that there can be no or only very little FRET between the chromophores within one E8 entity. Although some of the data may be interpreted as FRET within some of the observed entities, these rare observations, such as the increase of green fluorescence when the red fluorescence bleaches, can also easily be explained without the assumption of FRET as the underlying mechanism. In many cases we observed significant intensity fluctuations (increases and decreases) of pure red and pure green fluorescence from one recorded spectrum to the next, as in Fig. $3 \mathrm{a}$ where the green fluorescence at first decreases and then recurs after $3 \mathrm{~s}$. Furthermore it has been shown that the fluorescence rate of a single chromophore does not necessarily remain constant over time. Thus, we believe that the rare experimental observations which can be interpreted as an indication of FRET in fact reflect these intensity changes of independent emitters.

\section{Conclusions}

A fundamental question about the DsRed protein is the nature and composition of the oligomeric unit in solution and at a single molecule level. While ultracentrifugation experiments indicated that the protein forms an obligate tetramer in solution [5], it has not been possible using solution spectroscopy methods to elucidate the composition of the tetramer. A recent investigation by single molecule spectroscopy has addressed the question of tetramer composition [11]. We have used E8, a mutant of DsRed exhibiting both 
green and red fluorescence, to further investigate the factors influencing these details. E8 provides a convenient system free of the slow time-dependent spectral maturation seen in the wild-type molecule. Assuming that each monomer exhibits either green or red fluorescence, we find that E8 clearly forms hetero-oligomers, although it is not possible for us to determine whether these are dimers or other higher-order oligomers. Since the overwhelming majority of entities observed exhibit both green and red fluorescence, we must have at least dimeric species. Further, our data exhibit no evidence of fluorescence resonance energy transfer (FRET) between the green and red species in this DsRed variant. This could be due to unfavorable distances between donor and acceptor chromophores (unlikely), or to unfavorable orientations between the donor and acceptor dipoles. These observations are consistent with other spectrally resolved fluorescence lifetime microscopy measurements of E8 combined with photobleaching protocols that also do not indicate the presence of FRET (Subramaniam et al., in preparation). The detailed mechanisms underlying these observations remain under investigation.

\section{References}

[1] M.V. Matz, A.F. Fradkov, Y.A. Labas, A.P. Savitsky, A.G. Zaraisky, M.L. Markelov, S.A. Lukyanov, Nat. Biotechnol. 17 (1999) 969.

[2] D.L. Moon, M.G. Erickson, D.T. Yue, Biophys. J. 80 (2001) 362a.
[3] H. Mizuno, A. Sawano, P. Eli, H. Hama, A. Miyawaki, Biochemistry 40 (2001) 2502.

[4] S. Jakobs, V. Subramaniam, A. Schonle, T.M. Jovin, S.W. Hell, Febs Lett. 479 (2000) 131.

[5] G.S. Baird, D.A. Zacharias, R.Y. Tsien, Proc. Natl. Acad. Sci. USA 97 (2000) 11984.

[6] F. Malvezzi-Campeggi, M. Jahnz, K.G. Heinze, P. Dittrich, P. Schwille, Biophys. J. 81 (2001) 1776.

[7] A.A. Heikal, S.T. Hess, G.S. Baird, R.Y. Tsien, W.W. Webb, Proc. Natl. Acad. Sci. USA 97 (2000) 14831.

[8] B. Lounis, J. Deich, F.I. Rosell, S.G. Boxer, W.E. Moerner, J. Phys. Chem. B 105 (2001) 5048.

[9] M. Cotlet, J. Hofkens, F. Kohn, J. Michiels, G. Dirix, M. Van Guyse, J. Vanderleyden, F.C. De Schryver, Chem. Phys. Lett. 336 (2001) 415.

[10] M. Cotlet, J. Hofkens, S. Habuchi, G. Dirix, M. Van Guyse, J. Michiels, J. Vanderleyden, F.C. De Schryver, Proc. Natl. Acad. Sci. USA 98 (2001) 14398.

[11] M.F. Garcia-Parajo, M. Koopman, E.M. van Dijk, V. Subramaniam, N.F. van Hulst, Proc. Natl. Acad. Sci. USA 98 (2001) 14392.

[12] M.A. Wall, M. Socolich, R. Ranganathan, Nat. Struct. Biol. 7 (2000) 1133.

[13] D. Yarbrough, R.M. Wachter, K. Kallio, M.V. Matz, S.J. Remington, Proc. Natl. Acad. Sci. USA 98 (2001) 462.

[14] A. Terskikh, A. Fradkov, G. Ermakova, A. Zaraisky, P. Tan, A.V. Kajava, X. Zhao, S. Lukyanov, M. Matz, S. Kim, I. Weissman, P. Siebert, Science 290 (2000) 1585.

[15] J. Wiehler, J. von Hummel, B. Steipe, FEBS Lett. 487 (2001) 384.

[16] A.V. Terskikh, A.F. Fradkov, A.G. Zaraisky, A.V. Kajava, B. Angres, J. Biol. Chem. 277 (2002) 7633.

[17] B.J. Bevis, B.S. Glick, Nat. Biotechnol. 20 (2002) 83.

[18] H. Murakami, T. Hohsaka, M. Sisido, Nat. Biotechnol. 20 (2002) 76 .

[19] C. Blum, F. Stracke, S. Becker, K. Müllen, A.J. Meixner, J. Phys. Chem. A 105 (2001) 6983.

[20] T.A. Schuttrigkeit, U. Zachariae, T. von Feilitzsch, J. Wiehler, J. von Hummel, B. Steipe, M.E. MichelBeyerle, Chem. Phys. Chem. 2 (2001) 325. 